\title{
Porträt: Marian Chace
}

\author{
Cigdem Groll
}

$\mathrm{D}$ ie Pionierin Marian Chace wurde am 31. Oktober 1896 in Rhode Island (USA) geboren und prägte in ihrem 74-jährigen Leben nachhaltig die Tanztherapie.

Marian Chace begann ihre Karriere 1916 mit einem Studium für darstellende Kunst an der Corcoran School of Art. Ein paar Jahre später nahm sie Tanzunterricht. Bald begann sie auch schon mit ersten öffentlichen Auftritten, unterrichtete und choreografierte. Nachdem Marian Chace als Tänzerin bei der Denis-Shawn-Tanzkompanie und im eigenen Tanzstudio tätig war, entdeckte sie in den 1940er Jahren die Möglichkeit der Verwendung von Tanz und Bewegung in der Therapie (Wikipedia 2014).

Der authentische Gefühlsausdruck ihrer SchülerInnen rückte in den Fokus ihres Interesses und ihrer Aufmerksamkeit. In ihrer Arbeit widmete sie sich zunehmend therapeutischen Themen, die ihr wichtiger wurden als die Präzision und Optimierung der Tanztechnik. Entsprechend änderten sich auch die Ziele ihres Unterrichts. Die Inhalte ihres Schaffens entwickelten sich von der Tanztechnik zur Tanztherapie.

Die erste Gelegenheit, ihre Tanz- und Bewegungsarbeit patientengerecht weiterzuentwickeln und in die Praxis umzusetzen, ergab sich 1942 mit einem Auftrag am Elisabeth's Hospital in Washington, D.C. In einer Zeit, die offen war für neue Therapieformen, entwickelte sie ein auf Kommunikation und Körperwahrnehmung basierendes Programm für psychiatrisch hospitalisierte Menschen. Sie nannte

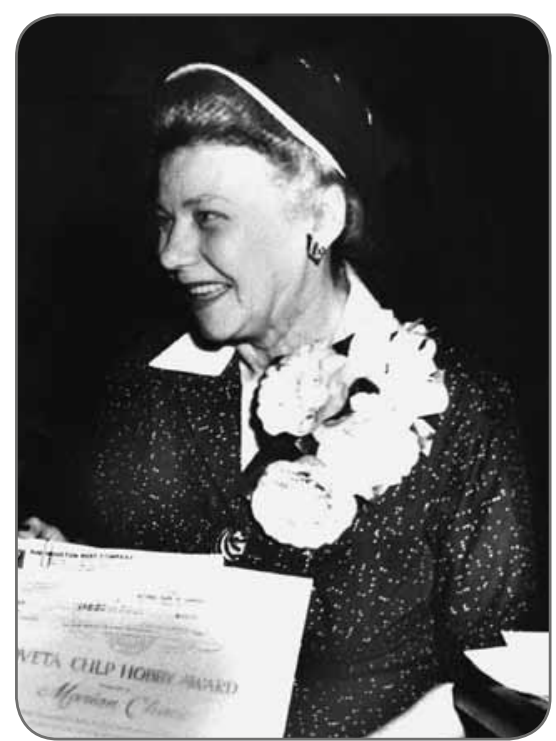

Marian Chace

Foto: Marian Chace Foundation (ADTA)

es „Dance for Communication“ oder „Social Dance“. Zu dieser Zeit waren Psychopharmaka noch nicht in die psychiatrische Therapie eingeführt worden (Institut am See für Tanztherapie 2008).

Marian Chace versuchte, Aggressivität und Unruhe der PatientInnen mit ihren methodischen Ansätzen des therapeutischen Tanzes zu besänftigen und in kreative Bahnen zu lenken (Willke et al. 1992). Sie konnte intuitiv erfassen, welche Bedeutung die soziale Interaktion in der Therapie hatte. In enger Zusammenarbeit mit Psychiaterlnnen und PsychologInnen entwickelte sie die theoretische 
Begründung für ihre Therapieformen. Später arbeitete sie in Chestnut Lodge / Rockville an einer Psychiatrischen Klinik, die bekannt war für ihre fortschrittlichen TherapeutInnen und innovativen Ansätze (Institut am See für Tanztherapie 2008).

Marian Chace ging in ihrem Konzept von folgenden Grundannahmen aus (Willke et al. 1992):

- Deformierungen der Haltung und der Funktion des Körpers waren ihrer Ansicht nach schlecht angepasste Reaktionen auf Konflikte. Der Tanz diente ihr als Mittel, um körperliche Verfestigungen aus Konflikten zu lockern. Dieser Prozess der Lockerung machte neue emotionale Erfahrungen möglich und sollte auf diese Weise zur psychischen Stabilisierung und der Auflösung von Hemmungen beitragen.

- Durch die Integration aller Gruppenmitglieder sollte das Selbstbewusstsein gefördert und das Ich gefestigt werden.

Auf diese Weise versuchte sie, den PatientInnen dazu zu verhelfen, ein realistisches KörperSchema-Empfinden zu entwickeln, Körperteile $z u$ aktivieren und in die Bewegungen zu integrieren, die Körperhaltung aufzubauen und zu korrigieren, selbstbestimmte Körperbewegungen wiederherzustellen und das Bewusstsein körperlicher Empfindungen zu verbessern (Institut am See für Tanztherapie 2008).

Marian Chace entwickelte dazu ihre eigene Methode und nutzte hierbei den Kreis als Raum für die Gruppe. Der Kreis stützte das gemeinschaftliche Gefühl der Sicherheit und Stärke. Die Gruppenmitglieder hatten hierbei ständig die Möglichkeit, Blickkontakt aufzunehmen, und wurden wiederum von den anderen wahrgenommen. Bewegungen der einzelnen Gruppenmitglieder wurden aufgegriffen, und es entstand ein gemeinsamer Rhythmus.

Marian Chace verwendete in der Bewegungsarbeit rhythmische Musik. Diese wirkte einerseits organisierend auf das individuelle Verhalten der PatientInnen und erzeugte andererseits Gefühle der Solidarität innerhalb der Gemeinschaft. Um diesen Prozess der Organisation und Gemeinschaftsbildung innerhalb der Gruppe zu ermöglichen, gliederte sie ihre Therapiesitzungen in einen charakteristischen Ablauf mit fünf Phasen:
Aufwärmphase
Spannungsabfuhr
Thema
Erdung
Abschluss

Die in der Gruppe vorhandenen Themen wurden durch dieses methodische Vorgehen sichtbarer, erlebbarer und damit auch einer therapeutischen Bearbeitung zugänglich. Die einzelnen Phasen wurden von Marian Chace angestoßen, begleitet und geführt, bis sie einfühlsam den Abschluss initiierte (Klein 1983).

Mit ihrer Methode stellte die Pionierin auf der Bewegungsebene eine therapeutische Beziehung her. Sie arbeitete dazu mit empathischer Interaktion, wodurch die Gefühlsqualität ihrer PatientInnen spürbarer und verständlicher wurde. Mit diesem Vorgehen nahm sie in ihrer therapeutischen Grundhaltung viele Merkmale der später von Rogers entwickelten personenzentrierten Methode vorweg (Institut am See für Tanztherapie 2008).

Sie vertrat die Ansicht, dass die Erfüllung grundlegender menschlicher Bedürfnisse das Wesen des Tanzes ist. Der Tanz befriedigt die Wünsche nach Kommunikation und erleichtert den Ausdruck von Gefühlen, ohne dass gesprochen werden muss.

Marian Chace war maßgeblich an der Gründung der ADTA (American Dance Therapy Association) im Jahre 1965 beteiligt, und noch heute zeigt sich in der Marian Chace Foundation, wie wichtig ihre Arbeit für die Tanztherapie bleibt. 1966 wurde sie die erste Präsidentin des nach wie vor einflussreichen amerikanischen Tanztherapie-Verbandes (Willke 2007). 
Ihr Einfluss und die von ihr entwickelte Methodik prägen nach wie vor die tanztherapeutische Arbeit im Kreis mit rhythmischer Musik und symbolischer Bewegung. Diese Vorgehensweise ist in der heutigen Tanz- und Bewegungstherapie ein wichtiger Bestandteil.

\section{Literatur}

Klein, P. (1983): Tanztherapie. Eine einführende Betrachtung im Vergleich mit Konzentrativer und Integrativer Bewegungstherapie. Dieter Balsies, Kiel Institut am See für Tanztherapie (2008): Tanztherapiegeschichte. Unterrichtsskript, Kesswil

Willke, E. (2007): Tanztherapie. Theoretische Kontexte und Grundlagen der Intervention. Hans Huber, Bern

Willke, E., Hölter, G., Petzold, H. (Hrsg.) (1992): Tanztherapie Theorie und Praxis - Ein Handbuch. Junfermann, Paderborn

Wikipedia (2014): Marian Chace. In: de.wikipedia.org/ wiki/Marian_Chace, 29.9.2014

\section{Anekdote}

Kommt eine Tanztherapeutin zum Zahnarzt.

Ganz um einen guten Kontakt zu seiner neuen Klientin bemüht, fragt dieser: „Und was machen Sie denn so beruflich?“"

Diese, ebenso schlicht wie präzise: „Ich bin Tanztherapeutin.“

„Ach ja“, strahlt der Zahnarzt, sichtlich stolz auf seine umfassenden Kenntnisse der therapeutischen Welt „das ist so etwas wie mit
Delfinen schwimmen!“, während er Spiegel und Bohrer in der Mundhöhle der Tanztherapeutin versenkt.

... nnngghhrrrra ...

Nach einer wahren Begebenheit.

Christine Schulz, Tanztherapeutin und Ausbilderin BTD 